\title{
Experimental Analysis of a Fully Compliant Grid Inverter with Controllable Response
}

\author{
Luis Reguera Castillo \\ Wind and Marine Doctoral Training \\ Centre \\ University of Strathclyde \\ Glasgow, UK \\ luis.reguera@strath.ac.uk
}

\author{
Dr. Antonio Lazaro Blanco \\ University Carlos III of Madrid \\ Leganes, Spain \\ alazaro@ing.uc3m.es
}

\author{
Dr. Andrew Roscoe \\ University of Strathclyde \\ Glasgow, UK \\ andrew.j.roscoe@strath.ac.uk
}

\begin{abstract}
This paper continues on from [1] where a grid connected inverter with ancillary functions was explained, designed and simulated. In this paper, the project will continue implementing the converter in a microgird; experiments have been conducted to evaluate the performance and fulfillment of a grid code compliant inverter. Before, the system had the capability of changing, automatically, the internal converter dynamics. Now, these functionalities have been exploited further and the it is able to adapt its response speed even when the system is already connected and injecting power to the grid. This characteristic makes the converter not only fully compliant with the most common grid codes of Spain, Denmark and the UK but also dynamic depending on the grid situation. As a result, the converter is more grid-friendly and easily customized and at the same time improves the stability of the converter itself.
\end{abstract}

\section{INTRODUCTION}

With the increased installation of converter-based system into electrical systems, stability problems have progressively steadily risen; countries with high converter connected generation such as UK, Ireland or Spain have begun to face technical difficulties [2], [3], [4]. In order to overcome these new challenges, the expectations of how grid connected converters operate are changing. If there is no fault or transitory effects on the grid, it is more convenient for the grid to make the converter control slower [5]. This makes the converter more robust to weak systems where the voltage quality of the grid to which the system is connected is poor, and the voltage transients are frequent. Due to the lack of speed on the control loop, the resulting control has an averaging effect over the events occurred on the voltage signal, responding to this in a much smoother manner than a faster, more nervous control. As a result, the system reacts like a system with a higher inertia, mitigating possible dangerous overshoots and swings in power that a faster control could provoke. This is further studied in [5] where an advised control bandwidth of less than $5 \mathrm{~Hz}$ is recommended to get a robust system.

On the other side, the so-called ancillary functions or grid supporting functions demand a fast response against events on the grid. As an example the Low Voltage Ride-Through curve is a common grid supporting function. It explains how the system has to react against a voltage drop to return the voltage to its nominal value, normally, injecting reactive power. As it can be seen in Fig. 2, the response required from the converter must be less than, between 0.15 and 0.6 seconds, depending on the country where the inverter is installed.

Without taking into consideration the difference in response time (this will be later discussed), it is clear that this requirement clashes with the one discussed previously where a $5 \mathrm{~Hz}$ of control bandwidth is advised.

In order to overcome this, converter systems are usually designed for the highest control bandwidth possible, allowing them to perform these ancillary functions very fast without major problems; however, as it has been explained before, this fast response would not be ideal. For the case where no events occur, the best performance possible would be to have a lower bandwidth; however, if any event is recognized, the ancillary functions will require a high one to respond with speed enough to fulfill the requirements.

In [1] a system with ancillary functions that has the capability of auto-designing its internal dynamics was presented and explained in high detail. For a defined crossover frequency $(F c)$ and phase margin $(P M)$, the regulators gains were calculated in a parameter file that is run before the control, and the converter, begin to operate. Therefore, this functionality was only available before the system was connected and injecting power to the grid. In this paper, this functionality will be further exploited, allowing the reconfiguration of the internal regulator gains dynamically by changing $P M$ and $F c$. If the control notices a change in these values, the regulator gains will be recalculated to satisfy the new dynamics. As a result, the converter will have flexible dynamics, ideal for both the grid and the converter.

Additionally, this plastic behavior is also very interesting from the converter manufacturer point of view. Since, as explained previously, the grid codes can vary immensely between countries (see Fig. 2); it should be necessary to set, in-situ, the regulator gains that satisfy the demanded dynamics for each grid code of each country. This high level design allows a much easier reconfiguration of, at least, the control 


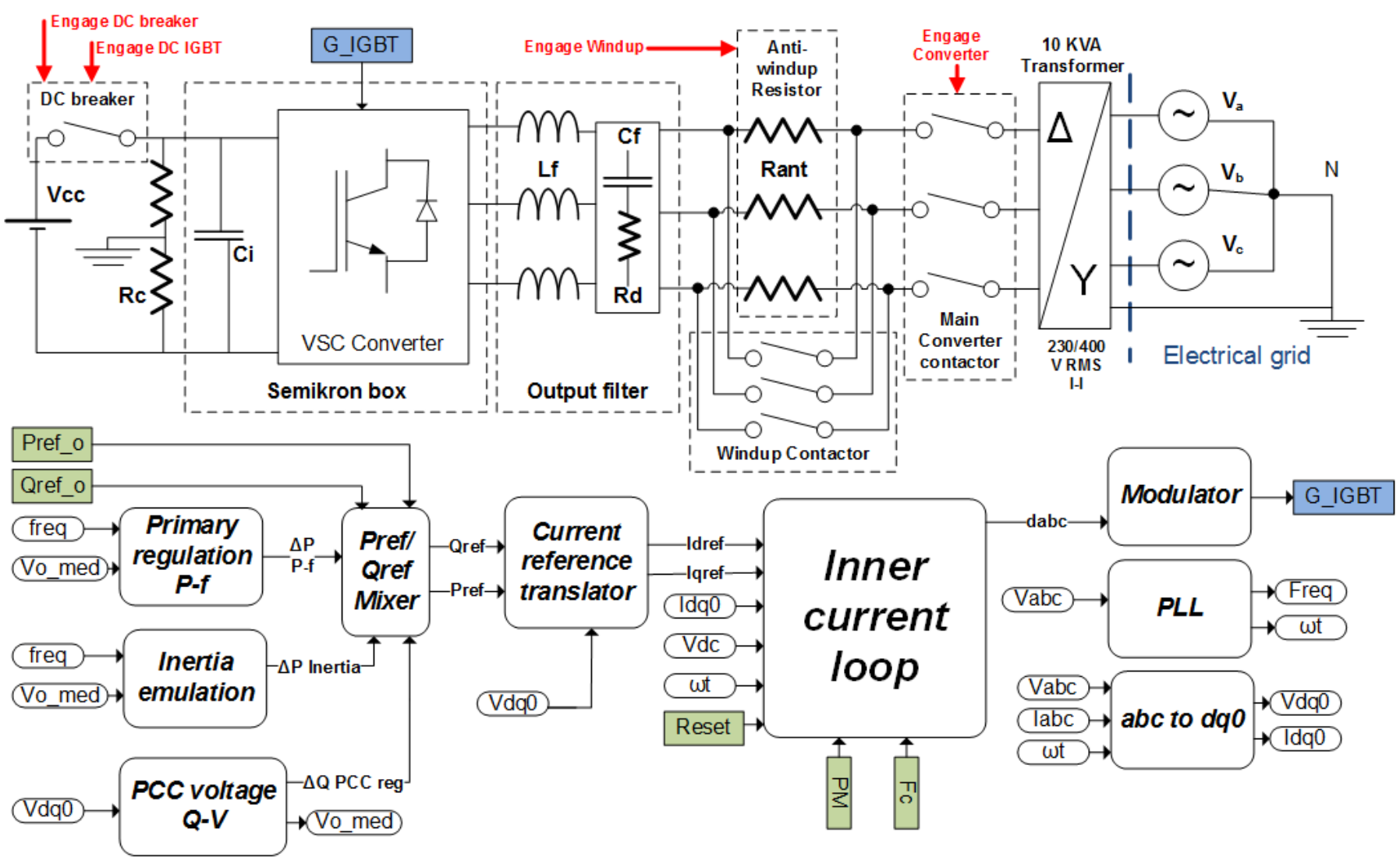

Fig. 1. Presentation of the system

part of the converter. Depending on the converter design and the required dynamics, it is highly likely that a change in just one or two parameters ( $F C$ and/or $P M$ ) from the parameters input set would allow a customizable response of the system that satisfies a pursued response.

\section{Presentation OF The System}

The converter consists a DC power supply of $600 \mathrm{~V}$ and 25 Amps, a Semikron SKS 25F converter which includes all the drivers for the IGBTs. Its output is filtered through a LC ( $L f$ and $C f$ ) filter connected in delta configuration. The capacitor

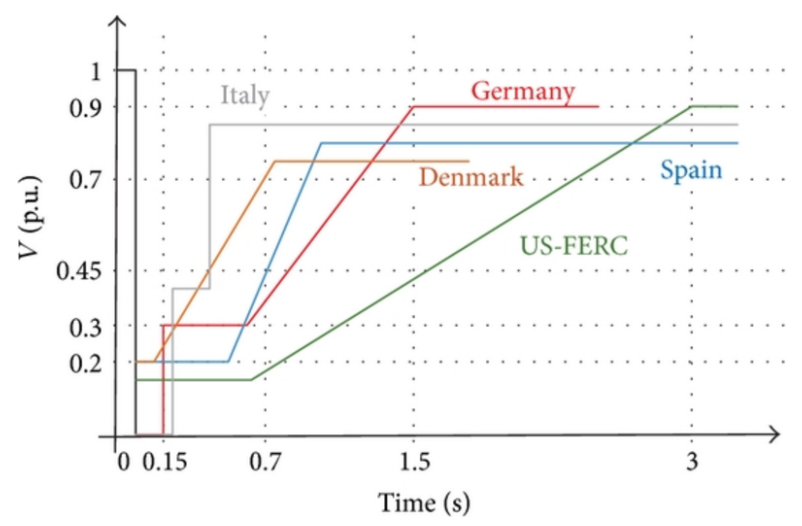

Fig. 2. Different LVRT curves for various countries [6] includes a small resistance in series to damp the resonance frequency of the filter $(R d)$. Following, an anti-windup resistor (Rant) is connected in series to provide a safe initialization of the system. Once the system is fully engaged, these resistors are bypassed by the contactor connected in parallel. Then, a contactor engages and disengages the converter. Finally, a delta-star transformer steps up the voltage to nominal level from 240 to 400 Vrms line-to-line. All the control is run in a dSPACE box based on the DS1006 processor card. Voltages and currents have been measured with sensors LEM LV-25P and LA-55P respectively. A table is attached in the appendix for the system values.

\section{A. Modifications from previous work}

As introduced previously, this paper continues on from the work done in [1]. Since the vast majority of the work remains intact, please refer to [1] for a detailed explanation of each block presented in Fig. 1. However, some modifications have been made since the system has been implemented in the lab. Therefore, and in order to improve the reproducibility of the system, all the changes will be detailed as followed:

The output filter in [1] was based on an inductance. In this paper the filter is upgraded to a LCL configuration, providing more rejection on the high frequency spectrum. In this case, the main DC power source is not a solar panel. Here, it is a DC power supply of $600 \mathrm{~V}$ and 25 Amps maximum. Since 


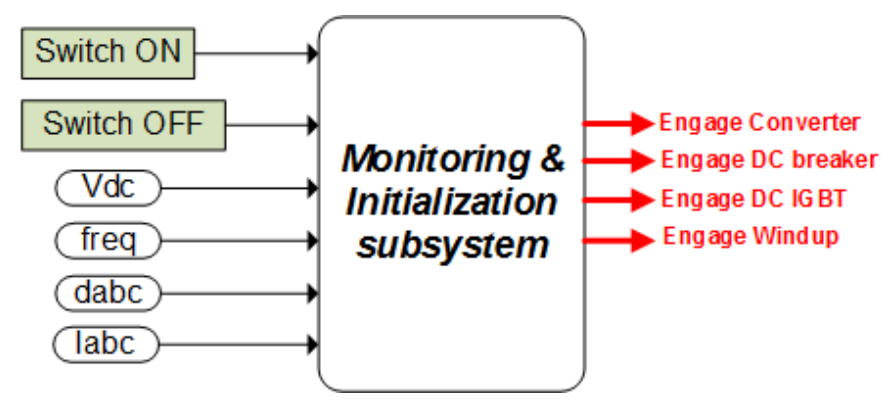

Fig. 3. Monitoring \& Initialization subsystem

the object of the paper is to exploit this dynamic capability of the converter control, this will not impact on the results obtained. Also, as there is no solar panel, there is no reason to implement the outer DC loop and the Maximum Power Point Tracker (MPPT).

The system now includes a new block that is not shown in Fig. 1 since it does not affect to the normal operation. This new block (shown in Fig. 3) is in charge of initializing the system safely, as well as continuously monitoring the system. If the system is exposed to dangerous situations where the integrity of the converter can be compromised, this block will disengage the system.

\section{B. Logic of control of the system}

Fig. 3 completes the logic of control with Fig. 1. Both Fig. 1 and Fig. 3, contain the input parameters of the system listed as following; in Fig. 1, the active (Pref_o) and reactive ( Qref_o) power references, a reset for the integral part of the regulator and $F c$ and $P M$ to change the speed response of the system; In Fig. 3, the switch on and off of the system. The only output of the system is in Fig.1 and corresponds to the IGBTs firing signals (G_IGBT) (shadowed in blue). Finally, contactor actions are in red. As it can be seen from Fig. 1, the logic of control is based on the standard vectorial current control. It measures the output currents and voltages and transforms them into the dq0 rotatory frame. This logic of control makes the converter able to provide a requested active and reactive power reference. Additionally, the ancillary functions (primary regulation, voltage control and synthetic inertia) outputs are combined modifying slightly the power reference values.

\section{Customizable response of the system}

Since this flexible behavior has already explained, please refer to [1] for a complete explanation. The contents explained in this section are just a summary.

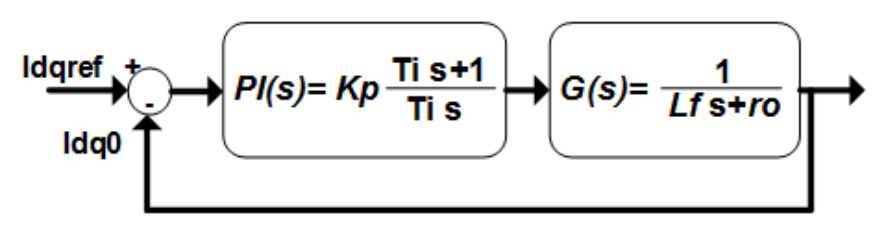

Fig. 4. Block diagram of the inner current loop
The method used here is based on the calculation of the gains of $T i$ and $K p$ of the PI regulator on the continuous domain. The plant to control the system is based on the output filter. Authors in [1] and [7] observed that the LCL filter plant can be approximated, for the regulation calculation purposes, as one unique equivalent inductance since it sets the dominant pole of the system that compromises the stability of the system. The plant with the regulator would then have the configuration presented in Fig. 4. Where $L f$ and $r o$ are the inductance and parasitic resistance of the filter ( $r o$ is not represented in Fig. 1 because it does not actually exist).

It is then possible to find $K p$ and $T i$ for a given set of $P M$ and $F c$ using the equivalent plant including the feedback loop and calculating the value of the resulting system in magnitude and angle for the demanded $F c(s=2 \pi F c)$ :

$$
\begin{gathered}
T i=\frac{\left(\frac{-\pi}{2}\right)+\left(P M \frac{\pi}{180}\right)+\operatorname{atan}\left(\frac{L f 2 \pi F c}{r o}\right)}{2 \pi F c} \\
K p=\frac{(T i 2 \pi F c) \sqrt{r o^{2}+(L f 2 \pi F c)^{2}}}{\sqrt{1+(T i 2 \pi F c)}}
\end{gathered}
$$

In [1] this calculation was done before the converter was connected to the system but in this paper, both equations have been included inside the logic of control. Therefore, every single time $P M$, or $F c$, are changed, a new set of $T i$ and $K p$ that satisfies these new values is calculated automatically and updated into the controller providing this dynamic behavior.

\section{EXPERIMENTS}

As it was initially introduced, the system has been implemented in the lab. The cited flexible behavior has been put into analysis and the results are presented in Fig. 5. The plots shown from top to bottom are as follows: phase voltages $(V a b c)$, current voltages $(I a b c)$, the active power reference and the real one extracted from the measured signals (Pref/Preal); the reactive power reference and the real one (Qref/Qreal), the phase margin $(P M)$, and the crossover frequency of the inner current loop $(F c)$. The experiment starts with the converter injecting $2 \mathrm{~kW}$ of active power with an initial crossover frequency of $100 \mathrm{~Hz}$. At approximately 2.1 seconds, a step of $2 \mathrm{KVAr}$ in the demand of reactive power from the transmission/distribution system operator side is executed. After approximately $200 \mathrm{~ms}$, the demand is satisfied and the converter injects a total of $2 \mathrm{~kW}$ of active power and 2 $\mathrm{kVAr}$ of reactive power. At 3 seconds the voltage falls to 0.85 pu (see Fig. 6), this is recognized by the internal logic and the system automatically reconfigures itself to fulfill the grid codes. In order to do so, a new reactive power step of $2 \mathrm{KVAr}$ is applied in Qref. Since this step comes from a grid supporting function, this response has to be faster than the previous command. Thus, automatically, the crossover frequency is increased to $300 \mathrm{~Hz}$. Internally, the gains from the PI regulators are recalculated and the reference is satisfied in a faster time than before. As a result, for the same step of reactive power $(2 \mathrm{kVAr})$, the former is much slower than the 

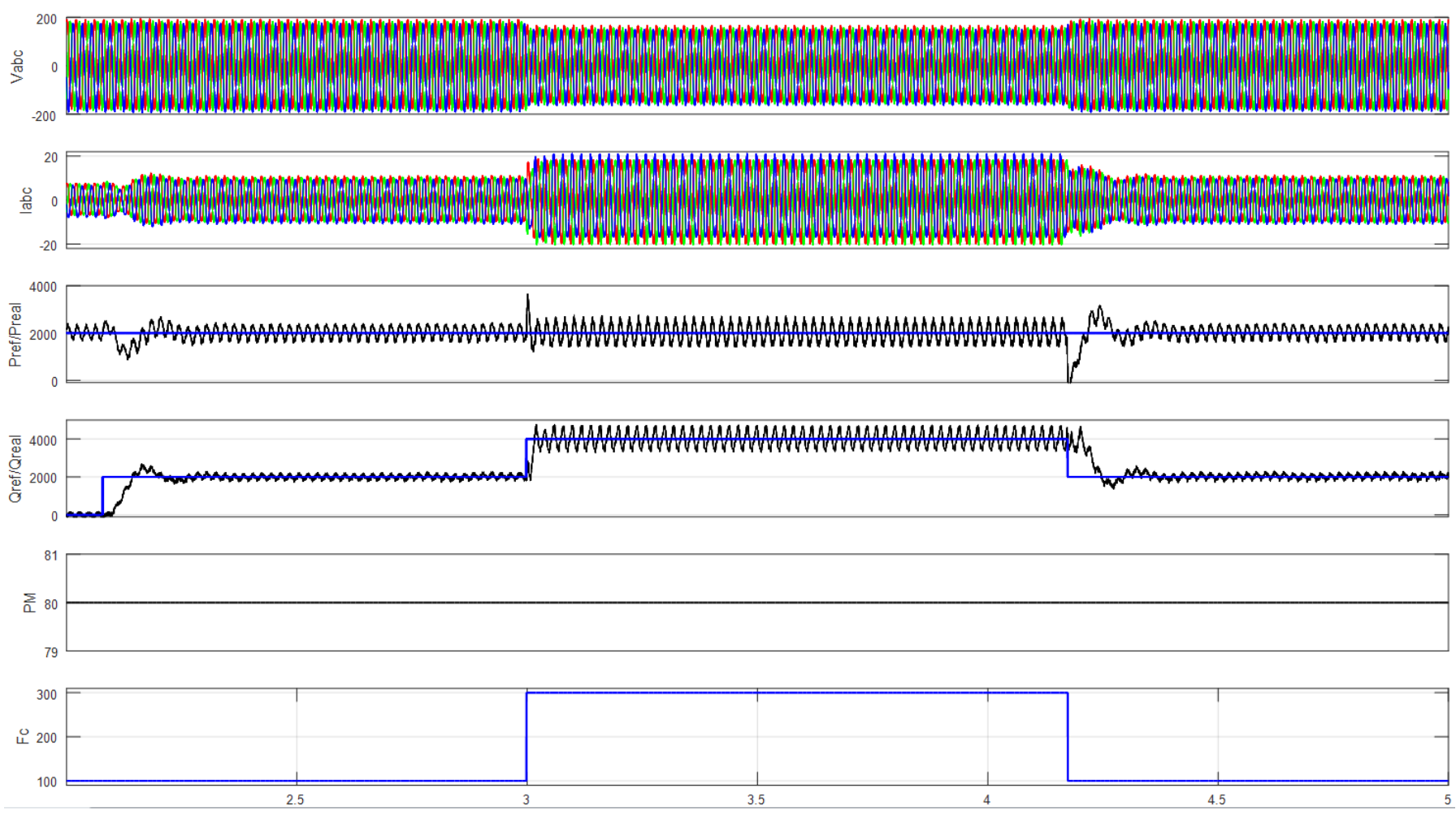

Fig. 5. Experimental results

latter, probing with it the conditioned behavior of the system depending on the events occurred on the grid.

It is important to remark that, from [1] and [4], it can be observed that this grid supporting function works with a lookup table and a gain to make the system respond proportionally to the voltage drop. In this case, for illustrations sake, this gain has been calculated in such manner that for a $15 \%$ voltage dip, an additional $2 \mathrm{KVAr}$ are injected $(\mathrm{Q}$ PCC reg $=$ $2 \mathrm{kVAr}$ ). Calculating the gain in this fashion makes it possible to perform a comparison between two equal steps of reactive power. One commanded by the operator, and other one by the grid supporting functions.

According to [8], it is required from the generators to respond against this event in less than $150 \mathrm{~ms}$. Since the first step takes $200 \mathrm{~ms}$ to be satisfied and the second $150 \mathrm{~ms}$, if $F c$ would not change from 100 to $300 \mathrm{~Hz}$ when it is necessary, this requirement would not be fulfilled. Therefore, the system would not be eligible for connection to the grid. Now, thanks to the customizable behavior, and without having changed any actual hardware in the system, the inverter responds $50 \mathrm{~ms}$ faster than before making it fully compliant with the regulation

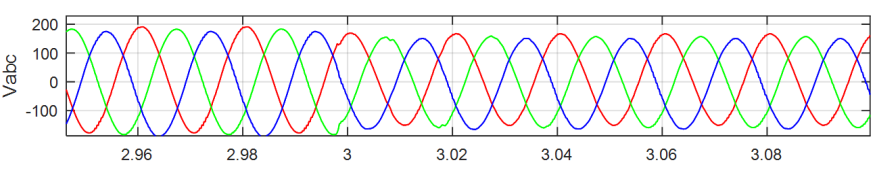

Fig. 6. Zoomed view of Vabc at 3 seconds established in the grid codes.

For simplicity, only the regulation of the PCC voltage function has been explained but, of course, the same procedure could be done with the remaining ancillary functions. Furthermore, a new set of $F c$ and $P M$ could be chosen for each ancillary function without any problem. This also would contribute to the philosophy established in this paper of flexible operation dependent on the outer conditions. Naturally, this flexibility will be limited, by the stability of the whole system.

\section{CONCLUSion}

A flexible and dynamic converter has been explained and demonstrated in this paper. Thanks to the tunable response of the system, the system can now be slow in the steady state regime and fast when there is any event on the grid. This dynamic response contributes to the stability and reliability of, not only the converter, but also the whole electrical grid, at the same time it makes the system more grid-friendly and tunable.

\section{REFERENCES}

[1] Reguera L., Lzaro A., Quesada I. and Barrado A., Auto-design simulation set up of PV VSC with grid supporting functions, IEEE 15th Workshop on control and Modelling for Power Electronics (COMPEL), Santander, 2014.

[2] EirGrid: 'DS3 Enhanced Performance Monitoring : Frequency Transient Analysis', EirGrid, 2013, https://tinyurl.com/h19xgzu , accessed March 2017.

[3] National Grid: 'Frequency Changes during Large Disturbances and their Impact on the Total System', National Grid, Proposal for consultation, https://tinyurl.com/z35vgpd, accessed March 2017 
[4] Spanish grid codes, Red Elctrica de Espaa (Transmission System Operator in Spain). https://tinyurl.com/hx94ovq (Consulted in March, 2017).

[5] Roscoe A.J., Yu M., Ierna R., Zhu J., Dyko A., Urdal H., Booth C. A VSM (virtual synchronous machine) convertor control model suitable for RMS studies for resolving system operator/owner challenges 15 th Wind Integration Workshop, Vienna, Austria, 2016.

[6] Yang Y., Blaabjerg F., Low-Voltage Ride-Through Capability of a SingleStage Single-Phase Photovoltaic System Connected to the Low-Voltage Grid, International Journal of Photoenergy, Feb 2013.

[7] M. Liserre, F. Blaabjerg and S. Hansen, "Design and control of an LCLfilter based three-phase active rectifier," Conference Record of the 2001 IEEE Industry Applications Conference. 36th IAS Annual Meeting (Cat. No.01CH37248), Chicago, IL, USA, 2001, pp. 299-307 vol.1.

[8] A. A. Rockhill, M. Liserre, R. Teodorescu and P. Rodriguez, "Grid-Filter Design for a Multimegawatt Medium-Voltage Voltage-Source Inverter," in IEEE Transactions on Industrial Electronics, vol. 58, no. 4, pp. 12051217, April 2011.

\section{APPENDIX}

\begin{tabular}{|l||l|l|}
\hline \multicolumn{3}{|c|}{ Experimental System values } \\
\hline$V c c$ & $600 \mathrm{~V}, 25 \mathrm{~A}$ & DC power source \\
\hline$S$ & $10 \mathrm{KVA}$ & Nominal power of the converter \\
\hline$R c$ & $10 \mathrm{~K} \Omega$ & $\begin{array}{l}\text { Input Resistance for DC bus discharg- } \\
\text { ing }\end{array}$ \\
\hline$C i$ & $2.2 \mu \mathrm{F}$ & Input Capacitor \\
\hline$I G B T$ & $25 \mathrm{~A}, 1200 \mathrm{~V}$ & Max Current, Max Vce voltage \\
\hline$L f$ & $3 \mathrm{mH}$ & Output filter inductor \\
\hline$C f$ & $8.8 \mu \mathrm{F}$ & Capacitor filter \\
\hline$R f$ & $22 \Omega$ & $\begin{array}{l}\text { LC Resonance frequency damping re- } \\
\text { sistor }\end{array}$ \\
\hline$R d$ & $100 \Omega$ & Antiwindup resistor \\
\hline $\begin{array}{l}\text { Output } \\
T R F\end{array}$ & $230 / 400 \mathrm{KVA}$ & Delta-Star connected \\
\hline$f s$ & $5 \mathrm{KHz}$ & Sampling and logic updating frequency \\
\hline
\end{tabular}

